\title{
URBANIZACIONES CERRADAS EN COSTA RICA: UN NUEVO OBJETO DE ESTUDIO ${ }^{1}$
}

\section{GATED COMMUNITIES IN COSTA RICA: A NEW OBJECT OF STUDY}

\author{
Alejandro Alvarado Alcázar* \\ Gustavo Jiménez Barboza**
}

"Al capitalismo salvaje le falló una variable dejó tanta gente afuera que ahora no puede salir..." Cristian Dzwonik (Nik) Historietista argentino

RESUMEN

La aplicación intensa del modelo neoliberal por toda América Latina a partir de la década de los 80 , ha traído consigo grandes transformaciones en sus estructuras sociales con una evidente expresión espacial en la ciudad, entre ellas, la emergencia de las urbanizaciones cerradas como hito inmobiliario de las elites de los países de la región. El presente artículo muestra el estudio del estado de esta nueva forma urbana en Costa Rica y los impactos que esta ha producido sobre la ciudad abierta.

PALABRAS CLAVE: SOCIOLOGÍA URBANA * URBANIZACIÓN * SEGREGACIÓN URBANA * URBANIZACIÓN CERRADA $*$ DESIGUALDAD SOCIAL

$1 \quad$ El artículo surge de la ponencia "Urbanizaciones cerradas en Costa Rica: impacto socio-espacial sobre los cantones de Escazú y Santa Ana 1990-2010" presentada en el VI Encuentro Nuevas Voces en Ciencias Sociales del Instituto de Investigaciones Sociales de la Universidad de Costa Rica, el cual tuvo lugar en la Sede Universitaria Rodrigo Facio, el 12 y 14 de abril de 2011. Es un proyecto de investigación de Trabajo Final de Graduación para la Licenciatura en Sociología.

* Equipo de Seguimiento y Análisis de la Coyuntura y la Conflictividad Social del Observatorio Social de América Latina (OSAL/IIS-UCR).

al.alcazar@hotmail.com

** $\quad$ Estudiante de la Licenciatura en Sociología de la Universidad de Costa Rica (UCR).

gustjim@gustjim.co.cc 


\begin{abstract}
The intense application of neoliberal model in all Latin America since the $80^{\text {th }}$, has brought with it great transformations on its social structures with an evident space expression in the city, among them, the rise of gated communities as a real estate milestone of the elite in countries of region. This article presents the study of the state of this new urban form in Costa Rica and the impacts they have had on the open community.
\end{abstract}

KEYWORDS: URBAN SOCIOLOGY * URBANIZATION * URBAN SEGREGATION * GATED COMMUNITY * SOCIAL INEQUALITY

\section{INTRODUCCIÓN}

En la actualidad uno de los temas más estudiados por la sociología urbana de la región, es el de la aparición de las urbanizaciones cerradas $^{2}$ como fenómeno urbano masivo (Thuillier, 2005). Para Roitman en “... la mayoría de los países, principalmente en los en vías de desarrollo, es un fenómeno que se ha manifestado en las ciudades más pobladas recién a partir de los 80" (2004: 6). La emergencia de este fenómeno es el producto de las profundas transformaciones que vivieron las sociedades latinoamericanas durante esos años en los que se dio la aplicación intensa del modelo neoliberal, provocando la modificación de la estructura productiva de los países de la región con la consiguiente modificación en las estructuras económica, social y política.

En el caso de Costa Rica, se podría afirmar que el estudio de las urbanizaciones cerradas y las consecuencias sociológicas que estas conllevan no ha sido abordado de modo sistemático, lo que implica un desconocimiento de dicho fenómeno. La única reflexión alrededor de esta nueva forma urbana en el país es la hecha por la historiadora Patricia Fumero, según la cual "las urbanizaciones cerradas han surgido como una respuesta ante los trastornos experi-

2 Siguiendo a Janoschka y Glasze, se entiende por urbanización cerrada a "... áreas residenciales que al menos contienen dos unidades (casas o apartamentos) físicamente separadas". Además que "... el área esté separada del resto del espacio accesible al público en general mediante un tipo de barrera física, algún tipo de servicio de uso comunitario o infraestructura $y$, además, que cuente con una organización vecinal" (2003: 10). mentados por la ciudad abierta como resultante de la mundialización de la economía y modelos de exclusión, desigualdad, aislamiento, fragmentación y multiculturalidad" (2009: 8-9). Si bien este trabajo resulta importante pues constituye un primer intento por problematizar el surgimiento de esta nueva forma urbana en el país, es necesario señalar que el análisis carece de una reflexión profunda que permita dimensionar el tema en su verdadera complejidad.

En el caso específico de este artículo, interesa recuperar el contexto en el cual surgen las urbanizaciones cerradas, atendiendo tanto las causas estructurales como individuales que propiciaron la emergencia de dicha forma urbana. De la misma manera, interesa presentar de manera suscinta algunos de los principales procesos de evolución urbana que ha seguido el Gran Área Metropolitana de Costa Rica hasta la actualidad, con el objetivo de recuperar la dimensión socio-histórica del fenómeno en cuestión. Asimismo, se discuten algunos de los presupuestos teóricos planteados por el marxismo para comprender la ciudad y los procesos que allí se desarrollan. Finalmente, se expone el caso del Condominio Santa Lucía en el distrito de San Juan de La Unión (Cartago), con la intención de presentar material empírico que contribuya a pensar un fenómeno aun no estudiado en el país.

\section{LAS URBANIZACIONES CERRADAS COMO FENÓMENO URBANO MASIVO}

La polarización social entre las clases sociales como producto del acentuado crecimiento de la desigual repartición de la riqueza, 
ha promovido una fragmentación social cada vez mayor, con una evidente expresión espacial en la ciudad. Es en este escenario donde se promueve que la burguesía y la pequeña burguesía de los países de la región migren hacia espacios caracterizados por romper con la noción histórico-negativa de la ciudad como un lugar caótico.

El incipiente desarrollo de esta nueva forma urbana en el país, la restringe a una función básica de hábitat, de residencia, de habitación; sin embargo, en torno a las urbanizaciones cerradas se han ido conformando anillos de bienes y servicios destinados a satisfacer las demandas que conlleva la instalación de este tipo de espacios en una zona determinada. A la par de las urbanizaciones cerradas, quizá el otro gran hito inmobiliario de las últimas décadas en el país, son los malls, los cuales, pese a que en un principio fueron ubicados por sus desarrolladores en áreas eminentemente urbanas, han comenzado a ser desarrollados, adaptando su crecimiento y ubicación en las áreas cercanas a estos nuevos espacios.

Esta situación, la proliferación de urbanizaciones cerradas, da origen a un fenómeno con profundas implicancias en la vida social de la ciudad. La segregación social urbana ${ }^{3}$ se convierte así en una característica inherente a la vida de este fenómeno, en la que la delimitación entre los espacios accesibles a todos y los accesibles solo a unos cuantos cobra su forma paroxística. Ahora bien, no puede considerarse que este fenómeno de la segregación sea exclusivo de las urbanizaciones cerradas, dado que desde el origen de la ciudad ha habido al menos una división simbólica entre explotadores y explotados, o como plantea Guy Thuillier: “... la distancia económica y geográfica entre las clases sociales no se inicia con los barrios cerrados" (2005: 16). Así, por ejemplo, en Costa Rica se puede pensar en Barrio Amón, Barrio Aranjuez y Barrio Escalante, como algunas de

3 Se entiende por segregación urbana "...la tendencia a la organización del espacio en zonas de fuerte homogeneidad social interna $y$ de fuerte disparidad social entre ellas, entendiéndose esta disparidad no sólo en términos de diferencia, sino de jerarquía" (Carballo, 2004: 11). las zonas que se convirtieron en residencia de la burguesía local desde finales del Siglo XIX, mientras lugares como Barrio México, Barrio Cuba y otros se constituyeron en los barrios obreros del país.

Un aspecto que de ninguna manera puede ser obviado en este análisis, es el hecho de que la proliferación de urbanizaciones cerradas tiene su expresión inversa en el mismo espacio y tiempo: los barrios urbano-marginales. Para Carballo:

En las ciudades latinoamericanas, en efecto, se da el proceso, por un lado, de la ilegalidad o precariedad de los asentamientos de una gran mayoría de la población, mientras que por otro lado, se consolidan las residencias de los sectores sociales privilegiados (2004: 11).

De un lado, parece que se reservan las mejores dotes de la modernidad, mientras del otro lado, solo se convive con lo peor de esta.

Las formas y funciones de las urbanizaciones cerradas están orientadas a satisfacer ciertas exigencias de los que se han visto beneficiados por el nuevo modelo de acumulación capitalista. Los sectores de la sociedad que tienen acceso a este tipo de espacios, ven en ellos la posibilidad de realizar su deseo de homogeneidad $y$ pureza social. Esto se ve reafirmado cuando se analizan las principales razones esgrimidas por quienes deciden migrar hacia urbanizaciones cerradas, varios estudios (Thuillier, 2005; Roitman, 2004; Arizaga, 2002 y 2003) concuerdan en que los principales argumentos son la calidad de vida y la seguridad que proveen este tipo de espacios. Es en este sentido, en el que se puede observar que existe un deseo evidente por borrar todo aquello nocivo que tiene la ciudad. El incremento en la inseguridad ciudadana y su correlato la afectación a la calidad de vida, son asumidos como males endémicos de la ciudad, por lo que quienes tienen la posibilidad optan por desplazarse hacia lugares en los que esto se ve invisibilizado por la presencia de barreras materiales, tales como, tapias, muros $y$ portones.

Todo parece indicar que se asiste a una nueva fase de fragmentación social de la ciudad, 
en la que la lógica instrumental y desgarrada se expresa en la polarización espacial entre zonas donde la opulencia es el pan de cada día y zonas donde el pan hace falta cada día. Una nueva morfología urbana comienza a aparecer en el país a partir de la proliferación de las urbanizaciones cerradas, con lo que el espacio urbano comienza a asemejarse cada vez más a otras ciudades latinoamericanas, en el que la privatización del espacio se torna una realidad cada vez más evidente.

\section{CRECIMIENTO URBANO Y PERIURBANIZACIÓN DEL GRAN ÁREA METROPOLITANA}

Para analizar el auge de las urbanizaciones cerradas en Costa Rica, se deben de tomar en cuenta varios aspectos históricos y socioterritoriales que se han dado a nivel urbano en el país, con la finalidad de comprender de una mejor manera la evolución del proceso. Para ello, en este apartado se mostrará la evolución del Gran Área Metropolitana (GAM) a partir de la década de los 50, así como, datos sobre la segregación residencial urbana, el escape de la burguesía y pequeña burguesía a los sectores este y oeste de San José y su posible relación con un proceso de periurbanización en la GAM.

A partir de los años 50, la zona urbana en Costa Rica experimentó un acelerado crecimiento como producto de la mejora en las condiciones de vida promovidas por el Estado Benefactor. En estos años, el aumento en la infraestructura estaba ligado al sector público para la prestación de servicios básicos ${ }^{4}$. Los emprendimientos privados estaban relacionados sobre todo con residenciales, industria y servicios comerciales (Pérez, 1998: 109). Entre el periodo de 1950 y 1980, el proceso de planificación urbana es prácticamente nulo y no existen reglamentos claros que indiquen la competencia en este aspecto del gobierno central, las

4 Para este periodo, Castro y Guido muestran que "... el Estado debía de participar activamente en la construcción de la infraestructura requerida para crearle las nuevas condiciones al capital, ya que se pretendía, en lugar de un proceso de centralización capitalista, uno de concentración con el propósito de formar nuevos capitales" (2000: 111). municipalidades y las instituciones autónomas $^{5}$. Es hasta 1979 que se crea el Ministerio de Vivienda y Asentamientos Humanos (MIVAH), el cual en el papel asume la tarea de regular la política urbana. Luego, a partir de la década de los 80, el país vive una crisis importante ligada a la crisis del petróleo mundial. En este periodo, el Estado disminuye la inversión pública y la población más vulnerable sufre un importante decaimiento en su calidad de vida. Las posibilidades de acceder a vivienda disminuyen, lo que provoca que se den varias tomas ilegales de tierras en varios lugares del país, principalmente en la GAM. Ya para finales de los 80 e inicios de los 90, el país alcanza cierta estabilidad económica mediante el endeudamiento externo, lo cual hace que los grupos empresariales ligados al sector inmobiliario se conviertan en importantes promotores en la construcción de urbanizaciones principalmente para los sectores socioeconómicos medios y altos. Es en este momento donde a partir de los Planes Reguladores se les da una participación más activa a las municipalidades en el proceso de Planificación Urbana. Esta descentralización de la política urbana en el país se da bajo las presiones de organismos internacionales como el Banco Mundial, el Programa de las Naciones Unidas para el Desarrollo, entre otros; provocando y facilitando que la planificación urbana se convierta en un espacio "... caracterizado por las corrientes que promueven la privatización y la desregulación como una especie de panacea para el desarrollo" (Retana, 2000: 101).

Ahora bien, uno de los principales aspectos que se debe de tomar en cuenta para el caso de las urbanizaciones cerradas es el de la segregación, ya que como señala Sabatini (2006), la segregación socio-espacial o socio-residencial no es solo de los pobres, sino también de los ricos. Para el caso costarricense, existe un estudio del Estado de la Nación en el que se indican importantes resultados que muestran la concentración de grupos de altos ingresos

$5 \quad$ Según Retana (2000: 98) sí se pueden observar en este periodo reglamentos como la Ley de Planificación Urbana (1968), el Plan de Desarrollo Urbano (1974) y el Plan Regional Metropolitano (1978). 
en sectores específicos de la $\mathrm{GAM}^{6}$, así como, los sectores de menores ingresos. Por ejemplo, muestran como los hogares de altos ingresos representan “... 24143 viviendas, un 4,9\% de las 498730 viviendas ocupadas de la GAM" (2005: 31). Entre los sectores de concentración de la burguesía y pequeña burguesía local encontramos a Escazú, Sabanilla de Montes de Oca, Belén, Rohrmoser, Sabana Oeste, entre otros. Contrastado con estos, los lugares de menores ingresos son La Carpio, San Felipe de Alajuelita, Sagrada Familia, Río Azul, etc. Este tipo de estudios muestran que a partir de la entrada del modelo neoliberal en el país, las crecientes desigualdades sociales han provocado que las clases más altas del país se muden hacia los sectores este y oeste de la GAM, tras convertirse San José en un lugar inhabitable para ellos.

En este mismo sentido, se muestran datos sobre el crecimiento en la inversión en

6 Esta distinción por ingresos lo hacen según la metodología de Necesidades Básicas Insatisfechas (NBI). vivienda en algunos de los cantones con concentración de grupos de altos ingresos y su contraste con la inversión en vivienda en los cantones donde se concentran los grupos de bajos ingresos.

Como se puede observar en el gráfico 1, en cantones como Escazú, Santa Ana y La Unión, se puede notar un creciente aumento de la construcción de urbanizaciones cerradas, sobrepasando los diez mil millones de colones, esto para los años de 2005, 2006 y 2007, mientras que San José y Desamparados, apenas sobrepasan la mitad de esa cifra para los mismos años. Para el caso de Alajuelita, se observa que no alcanza ni los cinco mil millones de colones para todos los años a partir de 1993. Estos datos indican que sectores del este (La Unión) y del oeste (Santa Ana y Escazú), son buscados por los grupos inmobiliarios para la construcción de viviendas lujosas, de alto costo económico y que buscan huir de los lugares "viciados y peligrosos" de la ciudad, como el centro de la ciudad (San José) y los barrios del sur (Desamparados y Alajuelita), sin quedar funcionalmente alejados de la ciudad central.

\section{GRÁFICO 1}

COSTA RICA: VALOR EN MILES DE COLONES DE VIVIENDA POR PERMISO PARA

ESCAZÚ, SANTA ANA, LA UNIÓN, SAN JOSÉ, ALAJUELITA Y DESAMPARADOS

1993-2007

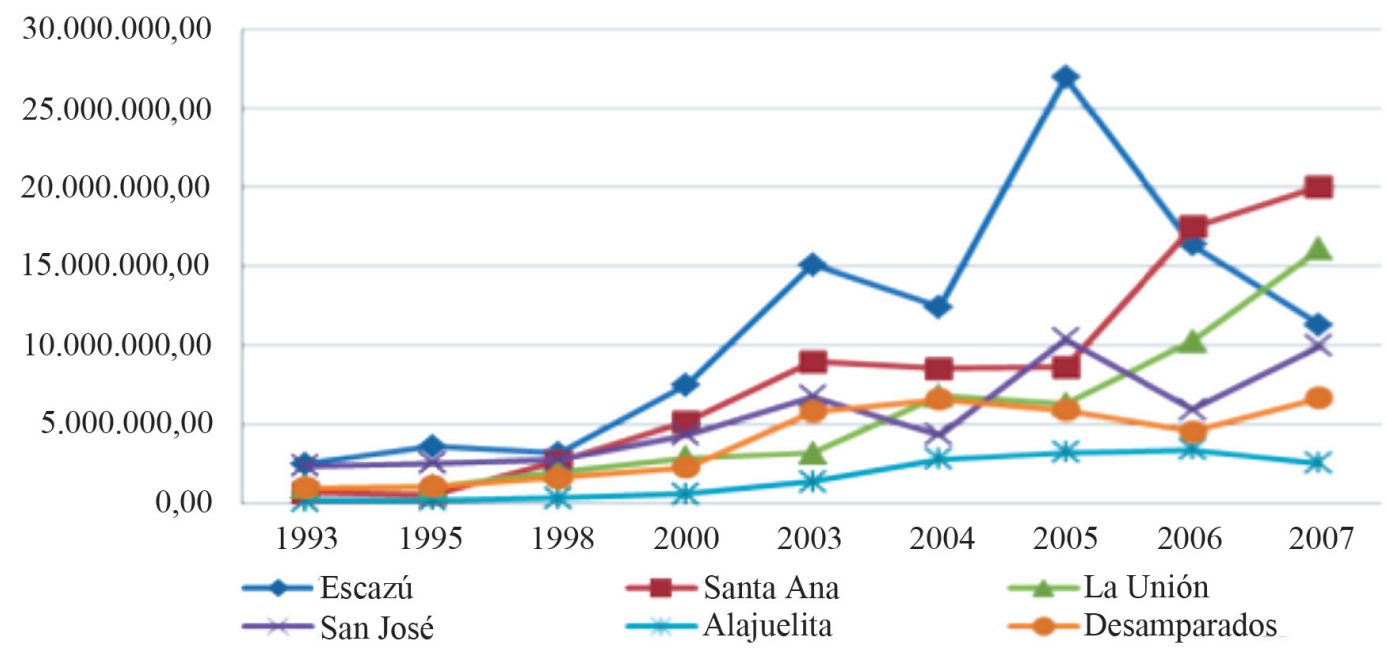

Fuente: Elaboración propia a partir de datos de Tendencias del Desarrollo Costarricense (http://www.tdc.odd.ucr.ac.cr/). 
La pregunta que queda es si estos lugares a los que huyen la burguesía y pequeña burguesía de nuestro país, están relacionados con el proceso de periurbanización de las regiones aledañas a la GAM. Primero que nada, se entiende por periurbano “... un área con constantes cambios en el uso del suelo, a la espera del avance urbano más cercano, sin la infraestructura apropiada para los desarrollos socioeconómicos que ya ocurren..." (Cardoso y Ortiz, s.f.: 2). Estas son zonas en las que antes se realizaban actividades como ganadería, agricultura, etc., y que en un momento determinado el capital cambia su uso de suelo en función de la obtención de aumentar la tasa de ganancia con respecto a la actividad anterior; por ejemplo, se puede ver que cantones como La Unión, funcionaban como grandes cafetales de sectores oligarcas y su uso de suelo fue cambiado sobre todo en función residencial y de abastecimiento (malls, servicios, etc.). Así, se observa que las regiones este y oeste, donde en mayor medida se ubican las urbanizaciones cerradas, son zonas que a partir de los años 90, han cambiado el uso de suelo de una gran proporción de su territorio en función residencial y de abastecimiento de servicios para elites económicas del país (nacionales y extranjeros), lo cual ha provocado una segregación socio-espacial claramente marcada material y simbólicamente. Se podría decir entonces, que los sectores a donde estas elites económicas se desplazan son zonas que están constantemente cambiando el uso del suelo, sobre todo en las dos funciones mencionadas.

\section{APROXIMACIÓN TEÓRICA AL OBJETO DE ESTUDIO}

Desde el punto de vista teórico, el fenómeno de las urbanizaciones cerradas representa un reto, ya que no cuenta con un cuerpo teórico propio para su estudio. Al respecto, Roitman señala que debido a su especificidad “... podría trabajarse con analogías ampliando perspectivas teóricas provenientes del estudio de otros fenómenos sociales o utilizando perspectivas teóricas ya existentes a este nuevo objeto de estudio" (2004: 11).
Es por esto que en este apartado se ensaya una propuesta teórica para su análisis, partiendo de las contribuciones del marxismo y complementadas por la discusión sobre la segregación urbana, en miras a construir un enfoque consistente para su estudio.

Los aportes a la sociología urbana de la llamada Escuela Marxista Francesa, principalmente los de Henri Lefebvre (1972, 1975a y 1975b) y Christian Topalov (1978), además de los de Manuel Castells (1977, 2001a y 2001b) ${ }^{7}$ parten de la crítica a la ideología urbanística, concibiendo lo urbano como una producción social en constante creación a partir de un proceso dialéctico del ser humano consigo mismo y con la naturaleza, el cual está atravesado por la lucha de clases y el papel que el Estado desempeña en dicho proceso. Castells (2001a) define la ciudad como un lugar geográfico en el que se instala la superestructura político-administrativa de una sociedad, la cual ha alcanzado un grado de desarrollo técnico y social tal que ha hecho posible una diferenciación entre la reproducción simple y ampliada de la fuerza de trabajo, con lo que se da origen a un sistema de repartición que supone la existencia de:

... 1) un sistema de clases sociales; 2) un sistema político que asegure a la vez el funcionamiento del conjunto social y la dominación de una clase; 3) un sistema institucional de inversión, en particular en lo referente a la cultura y la técnica; 4) un sistema de intercambio con el exterior (2001a: 43).

Este concepto es importante ya que permite la comprensión del fenómeno de la ciudad dentro de la lógica del capitalismo. Más allá de un concepto, es una lógica de dominación y explotación a partir de los aparatos ideológicos del Estado en miras de aumentar la tasa de ganancia. Además, esta ciudad debe de entenderse dentro de la lógica de la creación de toda una ideología alrededor de la cultura urbana.

$7 \quad$ Pese a ser de nacionalidad española, se incluyen los aportes de Manuel Castells dentro de la llamada Escuela Marxista Francesa, debido a su proximidad teórica con los autores mencionados. 
Esta tiene como fin la producción de una serie de valores sociales específicos, relaciones sociales y normas que son históricas, que poseen una lógica propia y que traen procesos de trasformación (2001b: 57). Uno de los mecanismos para crear esta cultura es la urbanización, la cual restringe a personas y funciones en un espacio determinado (Ibid.: 46), acá se da todo un proceso de densificación y heterogeneidad social (2001a: 50). A partir de Castells, se considera que las urbanizaciones cerradas se deben conceptualizar como un fenómeno de producción social de formas espaciales, el cual hace referencia al conglomerado que es "... funcional y socialmente interdependiente desde el punto de vista interno y en relación de la articulación jerarquizada (red urbana)" (Idem).

Para Topalov, el proceso de urbanización capitalista es la resultante de múltiples procesos privados de apropiación del espacio (1978: 20), si bien, esta afirmación se hizo pensando en un proceso más amplio - la industrialización- es aplicable al análisis de las urbanizaciones cerradas, pues ubica a este fenómeno en el escenario más amplio de los procesos que suceden en la ciudad abierta.

Una de las principales contribuciones del marxismo al estudio de los procesos urbanos, es que es una aproximación desde dos dimensiones: la histórica y la fenomenológica. El análisis crítico de lo urbano, señala Lefebvre, debe realizarse sobre dos dimensiones; de un lado, analizando las formas ideológicas, la cual refiere a los aspectos estructurales relacionados al cómo se distribuye lo urbano y el cómo se resuelven sus problemas, $y$ del otro lado, analizando las prácticas urbanas, es decir, las prácticas cotidianas de los individuos que intervienen en su producción.

A diferencia de otras corrientes teóricas, como la Escuela de Chicago, lo urbano es entendido como un espacio donde se dan formas de producción y reproducción social, las cuales se expresan espacial y temporalmente 0 , como señala Lefebvre “... espacial en la medida en que el proceso se efectúa en el espacio, al cual modifica por otra parte; temporal, puesto que se desarrolla en el tiempo..." (Ibid.: 13). Dicha conceptualización resulta central para el estudio de las urbanizaciones cerradas, pues se trata de un fenómeno reciente para cuya expli- cación es fundamental dar cuenta de las transformaciones acontecidas en las últimas décadas y cuya expresión en el espacio manifiesta cambios profundos en las estructuras sociales.

Así para Lefebvre, el estudio de los procesos urbanos se debe dar en tres niveles: a) el ecológico, que se encarga del hábitat y las formas en que se relacionan las personas; b) la fenomenología, que se encarga de la relación que existe entre ciudadanos y el medio y c) la morfología, que se encarga de lo que las personas hacen en la ciudad (1972: 54). Estos tres niveles ayudan a comprender las relaciones sociales capitalistas en las que se inserta el fenómeno de las urbanizaciones cerradas, así como, las funciones que estas cumplen dentro de la ciudad.

A partir de esta breve revisión, se quisiera establecer de qué manera las principales contribuciones hechas por la Escuela Francesa de Sociología Urbana son aplicables para el estudio de las urbanizaciones cerradas. A partir de la conceptualización de lo urbano como un producto social, en constante creación a partir de un proceso dialéctico de relación del ser humano consigo mismo y con la naturaleza, se considera que los niveles ecológico y la morfología de Lefebvre ayudan a comprender las relaciones sociales capitalistas en las que se inserta este fenómeno urbano, además de las funciones que estas cumplen dentro de la ciudad, por lo que es importante tenerlos en cuenta para la investigación. De Topalov se toma el tema de los procesos privados de apropiación del espacio, lo cual nos sirve de insumo para comprender la relación de la forma en que las empresas inmobiliarias diseñan espacios cerrados, en miras de la obtención de tasa de ganancia a través del uso y valor de suelo. Además, de Castells se incorpora el concepto de ciudad para comprender su lógica dentro el capitalismo, así como, el concepto de cultura urbana para entender los procesos ideológicos que se dan en torno a la urbanización (producción social de formas espaciales).

\section{CASO EJEMPLO: CONDOMINIO SANTA LUCÍA (SAN JUAN DE LA UNIÓN)}

Para ilustrar de manera preliminar las características principales de las urbanizaciones cerradas en el país, al no existir todavía un 
estudio específico al respecto, se muestra acá un material empírico para la discusión, el cual se presenta como un caso ejemplo basado en un trabajo de campo anterior realizado en el Condominio Santa Lucía ubicado en San Juan de La Unión, Cartago. Se considera oportuno relevar este caso para recuperar algunos de los aspectos desarrollados anteriormente, entre ellos los relacionados con las formas arquitectónicas y urbanísticas del lugar, las funciones y prácticas sociales, además de las vinculadas con las relaciones sociales que se establecen entre los residentes de esta urbanización cerrada y su entorno.

El proyecto residencial en estudio fue construido por la Urbanizadora La Laguna, la cual tiene a su haber proyectos tanto en los sectores este como oeste de la GAM. Una de las características de las urbanizaciones cerradas es la búsqueda de homogeneidad al respecto del resto de la ciudad heterogénea, o como lo plantea Thuillier:

... más todavía que las mismas formas urbanas, lo que diferencia a los barrios cerrados de los del centro de la ciudad $y$ del resto del suburbio es su homogeneidad, su coherencia arquitectural $y$ paisajística interna, garantizada por un grado muy fuerte de control comunitario sobre la producción del espacio urbano (2005: 10).

Para el caso del Condominio Santa Lucía, originalmente consistía en venta de lotes de $500 \mathrm{~m}^{2}$, en donde cada familia que compraba debía de enviar sus planos de construcción para ser aprobados por la administración. Los reglamentos de este proyecto urbanístico establecen que las casas no pueden tener más de dos pisos, que se prohíben algunas mascotas (tales como algunas razas de perros, reptiles, etc.), que no pueden existir muros internos, los colores de las casas deben de ser en tonos pasteles o terracotas. Asimismo, cualquier modificación después de terminada la casa debe ser llevada a la administración, donde es evaluada por un arquitecto de la Urbanizadora y varios vecinos miembros de un comité del proyecto, entre muchas otras $\operatorname{cosas}^{8}$. Esto diferencia a las urbanizaciones cerradas de la ciudad abierta, en cuanto logra controlar ("planificar") la forma en que esta se distribuye, en cómo debe de ser su forma.

Otra característica de esta nueva forma urbana es que se intenta recrear así un espacio que evoque a la comunidad, construyendo un ambiente suburbano que asegura el acceso a las dotes de lo tradicional y lo moderno, dejando de lado lo peor de los dos mundos (Arizaga, 2003: 16). La forma de venta con la idea o propuesta de una vuelta a la comunidad se puede observar en la publicidad con que se venden este tipo de proyectos. El caso de Santa Lucía, cuenta con amplias zonas verdes $y$ comunales como lugar de juegos para niños, rancho para actividades, cancha de tenis, sendero para caminar por las áreas verdes y en donde cada una de estas áreas cuenta con su respectiva reglamentación, que en caso de no ser cumplida es castigada con una multa. Asimismo, se cuenta con vigilancia las 24 horas del día, con dos guardas en la mañana y tres en la noche, los cuales están encargados de hacer una rigurosa inspección de las personas ajenas al proyecto, tanto cuando entran como cuando salen. Esto "asegura" que la "comunidad cerrada" sirva de modelo de antigua ciudad, en donde los "peligros no existían" y en donde los habitantes de la urbanización puedan estar tranquilos de que ellos y los suyos van a estar seguros de los peligros de la ciudad abierta.

Esta idea de la vuelta a la comunidad no parece cumplirse debido a que las relaciones entre vecinos son mínimas, ya que este tipo de urbanizaciones no hacen ver al individuo como un ciudadano o vecino, sino como un accionista dentro de un área de consumo (Thuillier, 2005: 16). Así, en el caso estudiado se puede percatar, que las relaciones que existen entre los vecinos son mínimas, ya que casi no se conocen $y$ algunos prefieren arreglar los conflictos por medio de intermediarios del proyecto, lo cual hace que el contacto cara a cara sea casi nulo.

$\overline{8} \quad$ En la actualidad, el proyecto cuenta con 59 viviendas que cumplen con las características mencionadas arriba sobre la cantidad de pisos, colores, etc. 
Todos los miembros del proyecto cuentan con carro y no es usual ver a las personas en las aceras del proyecto, salvo cuando pasean sus mascotas o hacen ejercicio. Además, la visibilidad a las afueras es muy poca, ya que sus verjas rodeadas de plantas omiten la visibilidad hacia la calle principal, tanto desde adentro como desde afuera. La visibilidad que existe es en la parte de atrás, en donde colinda con otra urbanización cerrada. Esto muestra que se quiere crear un sentimiento de homogeneidad social, en donde se modifica la relación con el otro ajeno a la urbanización, construyendo así un perfil casi único de accionista. Como lo menciona Rodríguez, la "Privacidad, aislamiento y cierres comparten protagonismo con una nueva sociabilidad de pequeños mundos cerrados pretendidamente homogéneos" (2004: 55). También, no solo las relaciones sociales a lo interno se modifican, al ser mínimas, sino que además con el afuera de la urbanización, ya que con los vecinos de los alrededores no se mantiene contacto constante. De modo que, las relaciones sociales que tienen las personas de estas urbanizaciones se dan a lo externo de la urbanización, en malls, trabajo, centros nocturnos, etc., por lo que la urbanización cumple una monofuncionalidad, la residencial.

Un aspecto fundamental que hace diferenciar la urbanización cerrada de la ciudad abierta, ya que como lo mencionan Lacarrieu y Thuillier

... en lo que más se diferencian las urbanizaciones cerradas de la ciudad abierta es en el grado de control comunitario sobre las formas $y$ usos del espacio urbano (...) precisamente para protegerse de esos usos no deseados del espacio urbano, las urbanizaciones privadas controlan muy estrictamente las prácticas desempeñadas en el espacio común, en realidad limitándolas al mínimo (2001: 90-91).

Esto se observa en el Condominio Santa Lucía, en tanto cuenta con una organización de los beneficiarios, la cual posee cédula jurídica y se reúne cada año para brindar informes, además de que se reúne extraordinariamente en caso de una eventualidad. Este tipo de control comunitario permite controlar cada movimiento que se haga dentro de la urbanización, jugando un papel de Estado que vigila, que no permite que ingrese "lo diferente". Así, se pudo constatar que esta organización se encarga de los cobros por motivos de multas, arreglos a la infraestructura, mantenimiento de áreas verdes $y$ recreativas; además, un tema importante es que se encarga de servir como mediadora, junto con la administración, en caso de conflictos entre vecinos, funcionando acá como un poder judicial.

En resumen, la urbanización cerrada no es solo un lugar en el que se quiera volver a la comunidad de antes, ni es solo paredes que resguarden de la "gran inseguridad" existente en el país, sino que se convierte en el lugar donde las relaciones personales se ven modificadas, tanto a lo interno como a lo externo, en ámbitos como el amiguismo, las relaciones vecinales, las relaciones de consumo, las formas de utilizar el espacio, etc. Esta parte del trabajo intenta ser exploratoria, ya que no se cuentan con datos y experiencias necesarias para exponer más sobre las urbanizaciones cerradas en el país. Desde los aspectos más estructurales de la ideología urbanística, así como, los más relacionados con la vida cotidiana se puede tener una visión crítica de este tipo de forma urbana a partir de las condiciones del capitalismo actual.

\section{CONSIDERACIONES FINALES}

Este trabajo tiene un alcance apenas exploratorio de las implicaciones que tiene la proliferación de las urbanizaciones cerradas en el espacio urbano en Costa Rica, así como, la posible relación que este hecho guarda con el aumento en la desigual distribución de la riqueza. Pese a esto, se considera que arroja pistas importantes para futuras elaboraciones acerca de un fenómeno que podría considerarse como el principal hito inmobiliario para las desarrolladoras, las cuales han visto en esto una gran fuente de extracción de ganancias.

A pesar de que en Costa Rica, el desarrollo de esta nueva forma urbana puede considerarse como reciente, todo parece indicar que 
continuará en desarrollo hasta transformarse en el hito inmobiliario de la burguesía y la pequeña burguesía local. En la región latinoamericana, el caso quizá más emblemático es el de la ciudad de Buenos Aires (Argentina), en la que en un radio de entre 30 y $60 \mathrm{~km}$ del centro de la ciudad se cuentan más de 400 urbanizaciones cerradas, que incluyen ocho proyectos de ciudades privadas ${ }^{9}$ (Vidal-Koppmann, 2004). En el país, las zonas periurbanas se han transformado en los lugares que dan albergue a la nueva y a la vieja clase dominante, pasando así de ser zonas boscosas, principalmente cafetaleras, a urbanizaciones cerradas en las que se restringe el tránsito de personas indeseadas. La realización del sueño impoluto de estos sectores se ve expresada espacialmente en estos proyectos, en el que las patologías de la vida urbana se ven desplazadas y reservadas para aquellos sectores que no tienen a su alcance el nuevo paradigma de la calidad de vida.

Para el geógrafo urbano, Luis Felipe Cabrales Barajas:

El interés por analizar la ciudad cerrada tiene el sentido de hacer visibles nuevos procesos de segregación social, fragmentación espacial, incremento de las distancias sociales $y$ evidenciar el reforzamiento de modelos que favorecen el repliegue de espacios públicos y entronizan las soluciones privadas al intentar resolver asuntos públicos (s.f.: 1).

Actualmente, en el país se vive la retirada del Estado en términos de la intervención y definición de políticas públicas y un realce de los intereses privados en los distintos ámbitos de la vida social. Es en este contexto en el que surgen fenómenos como las urbanizaciones cerradas, signados por la prácticamente absoluta autonomía del capital privado en la determinación de las características espaciales de las mismas, con lo que el interés público y colectivo se ve desplazado por los intereses privados e individuales.

$9 \quad$ Las ciudades privadas, conocidas comercialmente como ciudad-pueblo o pueblo privado, son proyectos concebidos para albergar a poblaciones de entre 20000 y 130000 habitantes.
El caso del condominio Santa Lucía en San Juan de La Unión en Cartago, sirve de material empírico para el estudio de las urbanizaciones cerradas en el país, pues como se señalo anteriormente, dicho fenómeno no ha sido estudiado. Como se pudo observar en el pequeño excursus analítico dedicado a la visita que fue realizada al lugar, las dinámicas de estos espacios son particulares y suponen la ruptura con múltiples características de la vida urbana. Lo que caracteriza la elección de ir a vivir a una urbanización cerrada es la búsqueda de un lugar menos caótico que la ciudad, más homogéneo, que vuelve a reencontrarse con las áreas de senderos, verdes y recreativas que abogan por la unión del núcleo familiar y el retorno a la comunidad (Thuillier, 2005: 19).

\section{BIBLIOGRAFÍA}

\section{LIBROS}

Carballo, Cristina. Crecimiento y desigualdad urbana: implicancias ambientales $y$ territoriales campana, 1950-2000. 1ra. edición. Argentina: Editorial Dunken, 2004.

Castells, Manuel. La cuestión urbana. 1ra. edición. España: Siglo XXI editores, 1977.

Castells, Manuel. "La urbanización". La sociología urbana de Manuel Castells. Susser, Ida (ed.). España: Alianza Editorial, 2001a.

Castells, Manuel. "La ideología urbana". La sociología urbana de Manuel Castells. Susser, Ida (ed.). España: Alianza Editorial, 2001b.

Lefebvre, Henri. La revolución urbana. 1ra. edición. España: Alianza Editorial, 1972.

Lefebvre, Henri. De lo rural a lo urbano. 3ra. edición. España: Ediciones Península, 1975a.

Lefebvre, Henri. El derecho a la ciudad. 1era. edición. España: Ediciones Península, 1975b.

Pérez, Marian. "La gobernabilidad urbana y la estrategia centroamericana de desarrollo sostenible: el caso del Área Metropolitana de San José". Gobernabilidad urbana en 
Centroamérica. Lungo, Mario (comp.). Costa Rica: FLACSO-GURI, 1998.

Topalov, Christian. La urbanización capitalista. 1ra. edición. México: EDICOL, 1978.

REVISTAS

Castro, Silvia y Guido, Francisco. "Ciudades intermedias en Costa Rica. Elementos socioantropológicos para su estudio". Revista de Ciencia Sociales 88 (II). Universidad de Costa Rica, abril-junio 2000: 109-122.

Fumero, Patricia. "La ciudad fragmentada: la Gran Área Metropolitana”. Herencia 22 (2). Universidad de Costa Rica, 2009: 7-12.

Janoschka, Michael y Glazse, Georg. "Urbanizaciones cerradas: un modelo analítico". Ciudades 59. Red de Investigación Urbana, 2003: 9-20.

Lacarrieu, Mónica y Thuillier, Guy. "Las urbanizaciones privadas en Buenos Aires y su significación". Perfiles Latinoamericanos 19. FLACSO, diciembre 2001: 83-113.

Programa Estado de la Nación. "Segregación residencial socioeconómica en la Gran Área Metropolitana”. Serie Aportes para el Analisis del Desarrollo Sostenible 10. Programa Estado de la Nación, agosto 2005: 31-66.

Retana, Juan Carlos. “¿Hacia dónde va la planificación urbana en Costa Rica? (Una interpretación sociológica)". Revista de Ciencias Sociales 88 (II). Universidad de Costa Rica, abril-junio 2000: 97-108.

Roitman, Sonia. "Urbanizaciones cerradas: estado de la cuestión hoy y propuesta teórica”. Revista de Geografía, Norte Grande 32. Pontificia Universidad Católica de Chile, diciembre 2004: 5-19.

Thuillier, Guy. "El impacto socio-espacial de las urbanizaciones cerradas: el caso de la Región Metropolitana de Buenos Aires". Revista EURE 93. 2005: 5-20.

TEXTOS ELECTRÓNICOS

Arizaga, María. "Nuevas urbanizaciones cerradas en los noventa: representaciones del suburbio en sectores medios". Documentos Jóvenes Investigadores 4. Universidad de Buenos Aires, junio 2003. En: <http:// www.iigg.fsoc.uba.ar/Publicaciones/JI/ ji4.pdf>

Cabrales Barajas, Luis. "Estado del conocimiento sobre la urbanización cerrada en Iberoamérica". Universidad de Guadalajara. En: <http://www.uib.es/ ggu/pdf_VII\%20COLOQUIO/16_CABRALES_ estadodelconocimiento.pdf>

Cardoso, André y Ortiz, Julia. "Periurbanización, segregación social y fragmentación territorial". Universidad Nacional de Tucumán. En: <http:// observatoriogeograficoamericalatina.org. $\mathrm{mx} /$ egal12/Geografiasocioeconomica/ Geografiaurbana/255.pdf>

Federación Interamericana de la Industria de la Construcción. "Evolución de la economía de los países miembros de la FIIC 20092010". Federación Interamericana de la Industria de la Construcción, 2010. En: <http://www.fiic.la/documentos/ Evolucion\%20Economia\%20Paises\%20 FIIC\%202009-2010.pdf>

Rodríguez, Isabel. "Urbanizaciones cerradas en Latinoamérica”. Ería 63 [Revista digital]. Universidad de Oviedo, 2004: 53-57. En: <http://dialnet.unirioja.es/servlet/ articulo?codigo $=977308>$

Sabatini, Francisco. La segregación social del espacio en las ciudades de América Latina. Banco Interamericano de Desarrollo, 2006. En: <http://idbgroup. org/sds/publication/publication_4338_s. htm>

Vidal-Koppmann, Sonia. "Archipiélagos urbanos en la periferia de la región metropolitana de Buenos Aires: las transformaciones socio-territoriales en la era de la globalización". Boletín del Centro Humboldt 11. Septiembre 2004. En: <www.flacso.org.ar/uploaded_files/ Tesis_Sonia_Vidal_Koppmann.pdf>

Fecha de ingreso: 10/05/2011 Fecha de aprobación: 01/11/2011 
